\title{
Validity of Self-reported Sexual Behavior Among Adolescents: Where Do We Go from Here?
}

\author{
Ralph J. DiClemente ${ }^{1,2}$
}

Published online: 12 October 2015

(c) Springer Science+Business Media New York 2015

\begin{abstract}
Adolescents have high rates of sexually transmitted infections (STIs). Adolescents consuming alcohol and using drugs have markedly greater HIV/STI risk and are a priority population for intervention. Accurate measurement of sexual risk behavior is critical for understanding individual's risk for HIV/STI, transmission dynamics of HIV/STI, and evaluating the efficacy of interventions designed reduce HIV/STI risk. However, significant challenges to accurately measuring adolescents' self-reported sexual behavior are well-documented. Recent advances in microbiology, such as the use of less invasive specimen collection for DNA assays, can assist researchers in more accurately measuring adolescents' sexual risk behavior. However, the majority of studies of adolescents' sexual risk rely solely on self-reported behavior; therefore, methods to improve the validity of adolescents' self-reported sexual behavior are needed. In addition, integrating biologic measures to complement self-reported measures are recommended, when appropriate and feasible.
\end{abstract}

Keywords Adolescents - Sexual risk behavior . Self-report · HIV/STI · Biological markers

Ralph J. DiClemente

rdiclem@emory.edu

1 Rollins School of Public Health, 1518 Clifton Rd., Atlanta, GA 30322, USA

2 Center for AIDS Research, Emory University, Atlanta, USA

\section{Validity of Adolescents' Self-reported Sexual Risk Behavior: Key Findings and Why It Matters}

Adolescents who use alcohol or drugs are at high-risk for HIV/STI acquisition and transmission. Thus, this is a priority population for interventions designed to reduce alcohol and drug consumption and/or enhance use of HIV/ STI risk-reduction practices. Accurate assessment of sexual behavior is critical for understanding risk for HIV/STI, HIV/STI transmission dynamics, and for evaluating the efficacy of behavioral interventions designed to reduce HIV/STI risk. A fundamental, and often neglected aspect of this research, has been the challenge of obtaining accurate self-report data about sexual risk behavior.

A number of studies have assessed the validity of adolescents' self-reported sexual risk behavior [1-4]. For example, in a nationally-representative school-based study, $10 \%$ of adolescents who tested positive for a sexually transmitted infection (STI) reported abstinence from sexual intercourse in the previous 12 months [4]. Similarly, another study found that $15 \%$ of men and $23.5 \%$ of women who reported always using a condom during sexual intercourse tested positive for an STI [2]. Such studies show marked discrepancies between self-reported sexual behavior and biologic markers assessing morbidity associated with sexual risk behavior.

Myriad challenges to accurate reporting of sexual behavior exist, including: recall bias, retrospective bias, socially desirable bias, low frequency of sexual behavior, low literacy, and poor comprehension of sexual behavior questions. In particular, alcohol and drug use may impair adolescents' recall of sexual risk behavior. Inaccurate reporting of sexual behavior, when randomly distributed makes it more difficult to detect intervention effects and when non-randomly distributed, produces bias which could 
lead to spurious conclusions. One key bias, social desirability bias, could result when participants in a risk-reduction intervention purposefully under-report their sexual risk behavior. Further, differential reporting related to alcohol and drug use, volume, or frequency may bias findings from studies designed to reduce HIV/STI risk among adolescent drinkers and drug users. Despite these thorny and problematic issues associated with self-report, most HIV/STI prevention intervention trials for adolescents have relied solely on self-reported sexual risk behavior to document programmatic efficacy (i.e., reductions in condomless sex). To gauge the extent of this problem we reviewed compendium of evidence-based interventions for adolescents (CDC's). Of the 20 programs identified, 16 $(80 \%)$ base their assertion of programmatic efficacy solely on self-reported measures of sexual behavior [5].

\section{Improving Validity of Self-reported Sexual Risk Behavior and Integrating Biologic Markers of Sexual Risk}

To more precisely quantify programmatic efficacy of HIV/ STI prevention interventions, there is a need to both improve the validity of self-report and to integrate biological markers of sexual risk behaviors into future adolescent HIV/STI risk-reduction intervention studies. A number of strategies have been recommended to improve the validity of self-reported sexual risk behaviors [6]. Some techniques include methods to improve recall by providing anchor dates, use of timeline follow back calendars, and/or recall of memorable events during a specified reporting period (i.e., previous three or 6 months). In addition, technological advances such as audio computer-assisted self-interviews may reduce socially desirable responding. Additional strategies to reduce socially desirable responding include placing the burden of denial on the participant (e.g. by reframing questions so that they ask "how many times" rather than "if" a participant engaged in a particular sexual behavior), making explicit researcher's efforts to keep participants' data confidential (e.g. use of identification numbers and secure data networks), and explaining that data will be analyzed across participants rather than by analyzing individual responses that could identify a particular participant. Other strategies for improving the validity of self-reported sexual risk behavior include using language that is easily understandable, stressing the importance of accurate reporting for the development of programs that may benefit others (altruism), and improving knowledge about sexual health and clarifying misunderstandings. In addition, consistency checks within surveys, feasible with the use of computer-assisted assessments, and across multiple assessments are other important methods to improve data quality [6]. It should be noted that inconsistent self-report of sexual behaviors within an assessment is indicative of invalid reporting. It is also important to note, that even though self-reports may be reliable (are consistent within assessments or across assessments) they may still be invalid. Thus, reliable reporting does not guarantee valid reporting. This situation is much more difficult to detect and poses a more serious challenge to assessing the efficacy of HIV/STI interventions.

Use of biologic markers of sexual risk is recommended, when appropriate and feasible, to complement self-report of sexual behavior. Laboratory-confirmed biologic end points are objective markers that provide an indicator of the adverse sequelae of sexual risks (i.e., STI, HIV) that can be administered in conjunction with selfreported measures. Biologic markers that can be integrated into HIV/STI prevention trials include laboratoryconfirmed STIs, pregnancy, prostate specific antigen (PSA), and Y-chromosome polymerase chain reaction (Yc-PCR). However, while biologic measures represent an advance in measurement, they are not a panacea, being subject to a number of limitations. For example, PSA and Yc-PCR assess engagement in condomless sex within a recent and narrow window of time ( $48 \mathrm{~h}$ for PSA and 14 days for Yc-PCR). In addition, pregnancy and Yc-PCR as objective biomarkers are only applicable to females. Additionally, pregnancy and laboratory-confirmed STIs may underestimate the true prevalence of sexual risk behaviors as these biological outcomes may not result from every act of condomless sex. Furthermore, studies conducted in populations with low STI incidence or pregnancy or with short follow-up periods or small sample sizes may not yield an adequate incidence of biological outcomes, thus, there may be insufficient statistical power to meaningfully analyze data on new pregnancy or STIs. Despite these limitations, use of biologic measures to complement self-reported sexual behavior data, when feasible, are encouraged.

\section{Summary and Recommendations}

There is an urgent need for HIV/STI prevention interventions for adolescents, in general, and adolescents who use alcohol and other drugs, in particular. Given the time allocated to developing new HIV/STI risk-reduction interventions and the significant financial investment needed to develop, implement and test these interventions, it is critical to improve the validity of adolescents' self-reported sexual behavior and integrate biologic measures into studies to more accurately measure the programmatic efficacy of HIV/STI prevention interventions. 


\section{References}

1. Rose E, Diclemente RJ, Wingood GM, et al. The validity of teens' and young adults' self-reported condom use. Arch Pediatr Adolesc Med. 2009;163(1):61-4.

2. Zenilman JM, Weisman CS, Rompalo AM, et al. Condom use to prevent incident STDs: the validity of self-reported condom use. Sex Transm Dis. 1995;22(1):15-21.

3. Brown JL, Sales JM, DiClemente RJ, et al. Predicting discordance between self-reports of sexual behavior and incident sexually transmitted infections with African American female adolescents: results from a 4-city study. AIDS Behav. 2012;16(6):1491-500.
4. DiClemente RJ, Sales JM, Danner F, et al. Association between sexually transmitted diseases and young adults' self-reported abstinence. Pediatrics. 2011;127(2):208-13.

5. CDC. Effective interventions: HIV prevention that works. 2014. http://www.effectiveinterventions.org/en/Home.aspx. Accessed 4 Jun 2014.

6. Weinhardt LS, Forsyth AD, Carey MP, Jaworski BC, Durant LE. Reliability and validity of self-report measures of HIV-related sexual behavior: progress since 1990 and recommendations for research and practice. Arch Sex Behav. 1998;27(2):155-80. 